\title{
Targeting Apoptosis for Optical Imaging of Infection
}

\author{
Mathew L. Thakur ${ }^{1}$, Kaijun Zhang ${ }^{1}$, Bishnuhari Paudyal ${ }^{1}$, Devadhas Devakumar ${ }^{1}$, Maria Y. \\ Covarrubias $^{3}$, Changpo Cheng ${ }^{2}$, Brian D. Gray ${ }^{4}$, Eric Wickstrom ${ }^{2}$, and Koon Y. Pak ${ }^{4}$ \\ ${ }^{1}$ Department of Radiology, Thomas Jefferson University, 1020 Locust Street, Suite 359-JAH, \\ Philadelphia, PA 19107, USA \\ ${ }^{2}$ Department of Biochemistry Molecular Biology, Thomas Jefferson University, Philadelphia, PA \\ USA
}

${ }^{3}$ Bioimaging, Kimmel Cancer Center, Thomas Jefferson University, Philadelphia, PA USA

${ }^{4}$ Molecular Targeting Technologies, Inc, West Chester, PA USA

\begin{abstract}
Purpose-Infection is ubiquitous and a major cause of morbidity and mortality. The most reliable method for localizing infection requires radiolabeling autologous white blood cells ex vivo. A compound that can be injected directly into a patient and can selectively image infectious foci will eliminate the drawbacks. The resolution of infection is associated with neutrophil apoptosis and necrosis presenting phosphatidylserine (PS) on the neutrophil outer leaflet. Targeting PS with intravenous administration of a PS-specific, near-infrared (NIR) fluorophore will permit localization of infectious foci by optical imaging.
\end{abstract}

Methods-Bacterial infection and sterile inflammation were induced in separate groups $(n=5)$ of mice. PS was targeted with a NIR fluorophore, PSVue ${ }^{\circledR} 794$ (2.7 pmol). Imaging was performed $(\mathrm{ex}=730 \mathrm{~nm}, \mathrm{em}=830 \mathrm{~nm}$ ) using Kodak Multispectral FX-Pro system. The contralateral normal thigh served as an individualized control. Confocal microscopy of normal and apoptotic neutrophils and bacteria confirmed PS specificity.

Results-Lesions, with a 10-s image acquisition, were unequivocally visible at 5 min postinjection. At $3 \mathrm{~h}$ post-injection, the lesion to background intensity ratios in the foci of infection $(6.6 \pm 0.2)$ were greater than those in inflammation $(3.2 \pm 0.5)$. Image fusions confirmed anatomical locations of the lesions. Confocal microscopy determined the fluorophore specificity for PS.

Conclusions-Targeting PS presented on the outer leaflet of apoptotic or necrotic neutrophils as well as gram-positive microorganism with PS-specific NIR fluorophore provides a sensitive means of imaging infection. Literature indicates that NIR fluorophores can be detected 7-14 $\mathrm{cm}$ deep in tissue. This observation together with the excellent results and the continued development of versatile imaging devices could make optical imaging a simple, specific, and rapid modality for imaging infection.

Correspondence to: Mathew L. Thakur; mathew.thakur@jefferson.edu.

Conflict of Interest

M.L. Thakur, K. Zhang, B. Paudyal, D. Devakumar, M. Y. Covarrubias, C. Cheng, and E. Wickstrom have no conflict of interest. Drs.

Brian D. Gray and Koon Y. Pak are the employees of Molecular Targeting Technologies, Inc. which sells PSVue ${ }^{\circledR} 794$. 


\section{Keywords}

Infection imaging; PSVue ${ }^{\circledR} 794$; Optical imaging; Confocal microscopy; Apoptotic neutrophils

\section{Introduction}

Infection is ubiquitous and can be lethal. The most reliable modality for imaging infection today is the one in which autologous white blood cells are labeled with a suitable radionuclide, injected back to the patient, and whole-body scintigraphic images are obtained $[1,2]$. The most commonly used radionuclides for imaging infection today are In-111 and Tc-99m for planar or single-photon emission computed tomography imaging and 2-deoxy-2$\left[{ }^{18} \mathrm{~F}\right]$ fluoro-D-glucose for positron emission tomography imaging. These methods, although clinically successful, are considered inconvenient and time-consuming [2]. Radiolabeled monoclonal antibodies specific either for CD67 or CD15 receptors expressed on human neutrophils which permit the labeling of human neutrophils in vivo have neither appealed to physicians (CD67), nor to the US Food and Drug Administration (FDA) (CD15), for routine clinical applications. [3-5]. Additional techniques that will image occult infection by a direct injection of a radioactive compound have been sought for decades, but none have succeeded for an acceptable, long-term clinical practice [5, 6]. Exploring novel approaches therefore is still worthwhile as they may satisfy the unmet needs of decades.

The resolution of infection, in part, is associated with neutrophil apoptosis, leading to necrosis [7]. The apoptosis of neutrophils and other inflammatory cells is thought to be accelerated by reduction of prosurvival factors such as lipopolysaccharides and certain cytokines such as IL-1B. These factors result from the clearance or removal of other inflammatory stimuli [8]. Apoptosis of neutrophils or other inflammatory cells may limit the severity of sepsis [9].

Apoptotic and necrotic neutrophils are presented, in the outer leaflet, with phosphatidylserine (PS), which is ordinarily sequestered in the plasma membrane inner leaflet, and provides itself as an excellent target for localizing infectious foci [10]. Recently, we targeted PS presented in the outer leaflet of apoptotic or necrotic neutrophil in experimental abscesses in mice with Tc-99m Annexin- $\mathrm{V}$ which has a high affinity for PS. All foci of infection were unequivocally imaged [11]. Like Annexin-V, PSVue ${ }^{\circledR} 794$, a nearinfrared (NIR) dye, containing $\mathrm{Zn}^{2+}$-dipicolylamine coordination complex, has also been shown to have a selective affinity for membrane enriched in anionic PS in such apoptotic cells, other than neutrophils, as Jurkat, CHO, HeLa, and Staphylococcus aureus [12, 13]. PSVue ${ }^{\circledR} 794$ is available commercially from Molecular Targeting Technologies, Inc., West Chester, PA, USA. The present investigation was initiated with a hypothesis that a NIR dye, PSVue ${ }^{\circledR} 794$, with its high PS affinity, will target apoptotic or necrotic neutrophil PS and will enable us to delineate experimental foci of bacterial infection and inflammation in mice by optical imaging.

Optical imaging is emerging as a sensitive, rapid, and high-throughput technology that permits longitudinal imaging to study a variety of cellular and molecular processes in vivo. Multiple applications of optical imaging in translational research are being evaluated [14]. 
An optical imaging approach in which apoptotic/necrotic neutrophils could be targeted with a PS-specific fluorophore injected directly into the patient's vein will, in principle, be a rapid, convenient, and a simple procedure for imaging occult abscesses and other inflammatory processes. This investigation is aimed at validating this hypothesis.

\section{Methods}

\section{Preparation of PSVue ${ }^{\circledR 794}$ Stock Solution}

A $1 \mathrm{mM}$ solution of PSVue ${ }^{\circledR} 794$, the structural formula of which is given in Fig. 1, was prepared according to the procedure provided by the supplier (Molecular Targeting Technologies, Inc., West Chester, PA). Briefly, a $2 \mathrm{mM}$ solution of pre-weighed apoPSVue ${ }^{\circledR} 794$ was prepared using vehicle $\mathrm{X}$ provided in a $2 \mathrm{~mL}$ solution. An equal volume of $4.2 \mathrm{mM}$ zinc nitrate solution was then added. The resulting solution was placed in a water bath at $40^{\circ} \mathrm{C}$ for 30 mins to ensure complete complexation. A resulting clear green solution of $1 \mathrm{mM}$ PSVue ${ }^{\circledR} 794$ was used.

\section{Inducing of Inflammation and Infection in Mice}

All procedures and animal protocols were approved by the Institutional Animal Care and Use Committee. Ten BALB/c mice, 5 to 6 weeks of age, were used. Subjects were selected at random for infectious or inflammatory lesions and injected with $100 \mu \mathrm{L}$ of bacterial culture or $50 \mu \mathrm{L}$ of turpentine, respectively. The concentration of bacterial cells was $1 \times 10^{8}$ cells per milliliter in saline and contained three strains: Escherichia coli, (gram-negative, ATCC-25922) Enterococcus faecalis (gram-positive, ATCC-29212), and Staphylococcus lugdunensis (gram-positive, ATCC-29213). It is our experience of several years that this cocktail induces pyogenic abscesses reliably $[6,11]$. Both infectious and inflammatory lesions were induced by intramuscular injection into the right thigh as per our protocol $[6$, 11]. Lesions were allowed to grow for 3 to 5 days, and initial assessment of their growth was determined by visual examination and palpation.

\section{Human Leukocyte Preparation}

Normal and apoptotic human leukocytes were used ex vivo to visualize the uptake of the fluorophore PSVue ${ }^{\circledR} 794$. Human leukocytes were isolated from $30 \mathrm{~mL}$ whole blood obtained via venipuncture, in $180 \mathrm{IU}$ of heparin, from healthy human volunteers. Whole blood samples were then mixed with $0.9 \mathrm{~mL}$ of $2 \%$ methylcellulose, sedimented at room temperature for an hour, leaving leukocyte-rich plasma (LRP) in the upper layer. LRP was separated from the sample, divided into two equal portions, and centrifuged for $5 \mathrm{~min}$ at $450 \times g$. The supernatant plasma was removed from the cells. One milliliter of $0.9 \% \mathrm{NaCl}$ in deionized water was added, and the cells were suspended gently and centrifuged for $5 \mathrm{~min}$ also at $450 \times g$.

\section{Ex Vivo Assessment of PSVue ${ }^{\circledR 794}$ Binding to Normal and Apoptotic Leukocytes}

Isolated human leukocytes were resuspended either in $1 \mathrm{~mL}$ autologous platelet-poor plasma (control) or to induce apoptosis, in $2 \%$ hypertonic saline, for $22 \mathrm{~h}$ in an incubator at $37^{\circ} \mathrm{C}$, containing 5\% $\mathrm{CO}_{2}$ and $95 \%$ air [11]. After the elapsed time, cells were centrifuged for 5 $\mathrm{min}$ at $450 \times \mathrm{g}$ and resuspended in $0.9 \%$ saline, and $5 \mu \mathrm{g}(2.7 \mathrm{pmol})$ of the PSVue ${ }^{\circledR} 794$ 
fluorophore was added. A similar quantity of the fluorophere was also added to an equal number of normal WBC in plasma $\left(20 \times 10^{6}\right)$ used as control cells. The cells were then incubated for $30 \mathrm{~min}$ in an incubator at $37^{\circ} \mathrm{C}$ containing $5 \% \mathrm{CO}_{2}$ and $95 \%$ air.

\section{Ex Vivo Assessment of PSVue ${ }^{\circledR 794}$ Binding to Normal and Apoptotic Microorganism}

Approximately $10^{8}$ Escherichia coli (gram-negative, ATCC-25922) in $1 \mathrm{~mL}$ culture medium was centrifuged each in two separate test tubes at $450 \times \mathrm{g}$ for $20 \mathrm{~min}$. The supernatant was discarded, and the microorganism was suspended in $1 \mathrm{~mL} 2 \% \mathrm{NaCl}$ and incubated at $37^{\circ} \mathrm{C}$ for $2 \mathrm{~h}$ to induce apoptosis. The bacteria were then centrifuged and resuspended in $1 \mathrm{~mL}$ culture medium. In a second test tube, $10^{8}$ E. coli was suspended in $0.9 \% \mathrm{NaCl}$. Approximately $10^{8}$ E. coli in $1 \mathrm{~mL}$ culture medium in a third test tube served as a control.

Five micrograms of the fluorophore was then added to each of the three test tubes and incubated for $30 \mathrm{~min}$ at $37^{\circ} \mathrm{C}$. In a separate set of experiments, gram-positive $S$. lugdunensis (ATCC-29213) at the same concentration were also processed and treated similarly.

\section{Confocal Microscopy}

Following incubation, white blood cells were centrifuged (450×g, $5 \mathrm{~min})$, the supernatant was discarded, and the cells were resuspended in $4 \mathrm{~mL}$ of respective medium, $0.9 \% \mathrm{NaCl}$ or autologous plasma. The white blood cells were then smeared (in duplicate) on glass slides, allowed to dry, and one slide from each cell type was incubated for $90 \mathrm{~s}$ with Wright's stain. The slides were rinsed with deionized water $(\times 3)$ and allowed to dry in the air. Following incubation with the fluorophore, both E. coli and the Staphylococcus were also smeared and stained similarly. A cover slip was then mounted, and each slide was observed using a Zeiss LSM 510 UV META confocal microscope (Carl Zeiss, Thornwood, NY). Images were acquired using a Plan-APO $\times 63$ oil immersion lens at room temperature and the Zeiss AM 4.2 SP1 Software. Images were analyzed using MetaMorph V7 6.5. At green channel, the excitation was at $488 \mathrm{~nm}$ and emission was at $505 \mathrm{~nm}$. For red channel, the excitation was at $633 \mathrm{~nm}$ and emission at $754 \mathrm{~nm}$. We realize that these wavelengths were not optimal for excitation $(e x \max =794 \mathrm{~nm})$ and emission $(\mathrm{em} \max =810 \mathrm{~nm})$ of PSVue ${ }^{\circledR} 794$.

\section{Optical Imaging}

Five mice in each of the bacterial infection and turpentine inflammation groups were administered $5 \mu \mathrm{g}(2.7 \mathrm{pmol})$ of the fluorophore in $150 \mu \mathrm{L} 0.9 \% \mathrm{NaCl}$ through a lateral tail vein. Although five mice can be imaged at one time in the Kodak Multispectral FX-Pro Imaging System (Carestream Health, Inc., Woodbridge, CT), for dynamic imaging, we imaged the five mice in each group one at a time, at $5 \mathrm{~min}, 15 \mathrm{~min}, 30 \mathrm{~min}, 1 \mathrm{~h}, 2 \mathrm{~h}$, and $4 \mathrm{~h}$ after injection. During imaging, each mouse was anesthetized by inhalation of $1.5 \%$ isofluorane and $98.5 \% \mathrm{O}_{2}$, and then placed in the supine position in the imaging chamber, maintained at $37^{\circ} \mathrm{C}$ and equipped with a controlled flow of $1.5 \%$ isofluorane and $\mathrm{O}_{2}$. The entire mouse was exposed to filtered light at $730 \mathrm{~nm}$, and $4 \times 4$ bit image was acquired for 10 $\mathrm{s}$ at $830 \mathrm{~nm}$. The CCD camera was set at $\mathrm{f}$-stop=2.4, focal plane, $2.5 \mathrm{~mm}$, and FOV $200 \mathrm{~mm}$ with no binning. 


\section{X-ray Imaging}

X-ray imaging was also performed at $1 \mathrm{MV}$ for a total acquisition time of $35 \mathrm{~s}$ immediately after optical imaging by placing a phosphor screen in the imaging field. The image capture setting was switched to X-ray imaging, and imaging was performed for $35 \mathrm{~s}$. The bright field images were captured and digitized.

\section{Image Preparation and Quantitative Analysis}

Multispectral FX-Pro software allowed the fluorescence and X-ray images to be coregistered and to perform quantitative image analysis of regions of interest. The analysis was performed on the infection/inflammation lesions and the clean, symmetrical, contralateral normal thigh muscles, which provided an internal control for each animal in the study protocol. The image intensity scale was kept constant, and the image intensity ratios were calculated for each time point image; mean \pm SD were calculated and plotted as a function of image time.

\section{Results}

The structure of PSVue ${ }^{\circledR} 794$, a NIR fluorescently tagged bis zinc (II)-dipicolylamine coordination complex, is given in Fig. 1. The turpentine inflammation was palpable within 3 days and the bacterial infection in approximately 4 days. Both the bacterial infection to background image intensity ratios and the turpentine inflammation to background ratios continued to increase for up to $3 \mathrm{~h}$ post-injection and then declined (Fig. 2). The longitudinal images obtained with a 10-s acquisition time of five bacterial infection-bearing mice and recorded at the same intensity are given in Fig. 3. The fluorescence images of all mice in each group clearly visualized the foci of infection consistently after 5 mins of injection of the PSVue ${ }^{\circledR} 794$. The relatively low uptake of the fluorophore in the liver, spleen, and kidneys had also cleared by $3 \mathrm{~h}$ post-injection, making it a most suitable time for imaging (Fig. 3). At $3 \mathrm{~h}$ post-injection, the infection to background ratio was $6.6 \pm 0.2$ as compared to that of $3.1 \pm 0.5$ for the inflammation (Figs. 3,4 , and 5). The uptake seen in the tail appeared to be due to infiltration of the fluorophore into the extravascular space. The uptake seen in the contralateral tibia appeared to be due to an external contamination from the excreted fluorophore as this uptake disappeared in those animals whose legs were washed with warm water.

Figure 6a demonstrates the confocal image of normal white blood cells and Fig. 6b, of those white blood cells that were incubated with the fluorophore. The absence of fluorescence in Fig. 6b indicates the lack of available PS in the healthy cells and that normal white blood cells do not stain with the fluorophore. In Fig. 6c, apoptotic white blood cells are visible. Figure 6d shows that these cells were heavily labeled with the NIR PSVue ${ }^{\circledR} 794$ and demonstrates the corresponding fluorescence due to targeted PS in these apoptotic cells. Figure 7a shows normal, gram-positive $S$. lugdunensis. Some dividing bacteria are visible. Figure $7 \mathrm{~b}$ shows the PSVue ${ }^{\circledR} 794$ fluorescence on some normal bacteria. Figure $7 \mathrm{c}$ represents apoptotic Staphylococcus and Fig. 7d, the corresponding microorganism with enhanced PSVue ${ }^{\circledR} 794$ fluorescence. Despite the suboptimal excitation and emission wavelength, intense fluorescence was visible in apoptotic white blood cells as well as in apoptotic 
grampositive Staphylococcus. With the gram-negative E. coli strain (ATCC-29213) we used, neither normal bacteria (Fig. 8a, b) nor their apoptotic counterpart (Fig. 8c, d) showed any fluorescence of PSVue ${ }^{\circledR} 794$.

\section{Discussion}

The present results provide unequivocal images of the infection and inflammation foci by a simple, single administration of the NIR fluorophore PSVue ${ }^{\circledR} 794$, which has a high affinity for PS exposed on the outer leaflet of apoptotic/necrotic cells. The abscesses were detectable in 5 mins after direct, intravenous administration of the fluorophore, although most intense images were seen at $3 \mathrm{~h}$ post-injection. Only $10 \mathrm{~s}$ was required for image acquisition.

Scintigraphic imaging of abscesses, either in experimental animals or in patients, have now been explored since 1977 [1, 15]. In all of those prior investigations, the experimental abscesses have never been visualized with such a short duration of image acquisition, or with such a short time after injection of an imaging agent, including those that targeted white blood cells in vivo [3-5]. The simplicity of its intravenous administration, the speed of its image acquisition, and the novel mechanism by which it visualizes infection in vivo make PSVue ${ }^{\circledR} 794$ a highly attractive and useful agent for imaging infection. The dose of $5 \mu \mathrm{g}$ (2.7 $\mathrm{pmol} / 25 \mathrm{~g}$ mouse) was chosen to avoid any possibility of auto-attenuation.

In addition, to examine the apoptotic neutrophils and other inflammatory blood cells that may have exposed PS on their outer leaflet for PSVue ${ }^{\circledR} 794$ targeting, we investigated the PS contribution by the microorganisms. Our data (Fig. 7a-d) suggest that gram-positive microorganisms do contribute to the fluorescence that permits imaging of the abscesses, but the gram-negative strain that we used did not.

Gram-negative bacteria have an extra layer on their cell wall which prevents them from being stained. The extra cell layer might be the reason by which the bacteria did not show any fluorescence after incubating them with the NIR probe. However, conflicting observations have been published, staining for one strain of E. coli [14], but not of the other [16]. The E. coli strain that we used (ATCC-29213) clearly did not interact with PSVue $^{\circledR} 794$.

Previously, the contribution of PS fluorescence arriving from apoptotic $S$. aureus in imaging bacterial abscesses is well demonstrated $[13,16]$. However, the contribution to the image intensity of PS fluorescence from apoptotic or necrotic white blood cells migrated to the bacterial infection was not investigated. Our data shown here clearly illustrate the intense contribution to the image intensity of PS fluorescence resulted from apoptotic/necrotic white blood cells as well as from gram-positive $S$. lugdunensis. In our experimental setting, however, the PS fluorescence resulted from E. coli was minimal. This may be due to the strain of bacterial we used in this study $[14,16]$. These data are consistent with our previous investigations in which we have successfully targeted apoptotic/necrotic white blood cell PS with Tc-99m Anexin V and unequivocally imaged bacterial infection in experimental mice. The data also validate our hypothesis that a PS-specific optical fluorophore may image abscesses. 
If we consider that the neutrophil PS fluorescence is the primary cause for imaging bacterial infection with high intensity (high infection/background ratio), then the relatively low intensity in the turpentine-induced inflammation imaging cannot be fully explained. In the inflammatory foci, the infiltration of neutrophils is equally high. It is possible, however, that the residual turpentine in the inflammatory foci may have quenched the fluorescence intensity. Turpentine quenching should not have any bearing on optical imaging of inflammation in humans. Carrageenan-induced experimental inflammation, although we did not try it, may eliminate the self-quenching possibility [17].

This approach to imaging abscesses, by specifically targeting the involved apoptotic neutrophils, with a directly injectable, non-radioactive fluorophore is not only simple but also rapid and appealing. It is appealing because it offers simplicity and eliminates the blood withdrawals, ex vivo cell separation, and radiolabeling. Optical imaging also eliminates the exposure to radiation for the staff as well for the patients. In principle, it can be used to image infection of various kinds and permits longitudinal imaging when called for, in the continued management of this disease.

Optical imaging is emerging as a powerful modality in molecular imaging which is applicable to better understand a variety of in vivo cellular interactions, including in the management of certain life-threatening diseases. However, at the present time, it produces significant challenges in imaging those lesions that are seated deep in the body, particularly in humans.

The real limitation of this approach to image abscesses therefore may stem from the lack of depth penetration of emitted light photons. For every centimeter of tissue thickness, the light signal intensity is reduced by ten times [18]. To image fluorescence deeper in a body, optical imaging near-infrared fluorescence (NIRF) with emission wavelength between $650 \mathrm{~nm}$ and $900 \mathrm{~nm}$ is the best approach available today in optical imaging. In this wavelength range, attenuation of light photons by water, lipids, and hemoglobin is minimal. Furthermore, muscle autofluorescence is also minimal. Some authors $[18,19]$ assert that NIRF can theoretically be detected at depths of 7-14 cm in tissue. Currently, however, most optical imaging instruments are largely confined to small animal imaging. Therefore, this theory cannot be validated experimentally in large animals such as rabbits or dogs. However, if such is the case, then many foci of infections can be detected in humans in the anterior or posterior positions. It appears that the challenges of imaging human whole body in a specially designed dark room may not be insurmountable, since the techniques such as diffuse optical tomography promise to image lesions nearly $20 \mathrm{~cm}$ deep in the body [19]. Given the success of clinically useful applications of NIRF imaging, hybrid and tomographic imaging with high spatial and temporal resolution may be plausible. Indeed, instrumentation for NIRF detection in humans is under development including the ART SoftScan, the optical breast imaging device from Advanced Research Technologies. This device provides functional information on the physiologic status of tissue using near-infrared light and is currently approved in Canada and some countries in Europe. It is undergoing the device approval process by the US FDA. The availability of such smart devices then will lead to additional and more exciting translational applications of optical imaging for the management of a wide range of diseases. 
Although optical imaging techniques are highly sensitive in that they require relatively only a small quantity of the fluorophores, their applications for human use must be approved by the FDA. One such NIR fluorophore, indocyanine green, has already been approved by the FDA for retinal angiography and to examine liver function [13].

At the present time, PSVue ${ }^{\circledR} 794$ optical imaging technique may be readily applicable in the management of such diseases as osteomyelitis colitis and inflammatory bowel disease. This may be achieved by insertion of a NIR excitation probe and a miniature imaging probe allowing external visualization of infection/inflammation following an intravenous administration of PSVue ${ }^{\circledR} 794$. Furthermore, the PSVue ${ }^{\circledR} 794$ can also be used to assess neutrophil viability after they have been manipulated externally for radiolabeling or separated and stored for transfusion.

\section{Conclusion}

Infection is ubiquitous and is a cause of millions of fatalities each year worldwide. Great strides have been made in radiologic methods for localizing foci or infection, but they all suffer from various limitations. Furthermore, the radiation dose delivered to the patient from CT or scintigraphic imaging methods prevent their use for repeated examinations that may be required for the management of infection. Our data indicate that application of the optical imaging methods for imaging infection deserves a serious consideration as it is simple, rapid, sensitive, exploits specific molecular biomarkers resulting from biological cell signaling pathways, and can be used for repeated patient examinations. It is hoped that the continuous and promising development in optical imaging devices will make optical imaging a method of choice for imaging infection and inflammation in the near future.

\section{Acknowledgments}

Dr. Mathew Thakur and Dr. Kaijun Zhang thank Dr. Donald Jungkind and Mr. David Lander for the supply of the microorganisms.

Research was supported by NIH/NCRR 1S10RR 026678-01 (MLT) and NIH 5P30 CA-56036.

\section{References}

1. Thakur ML, Coleman RE, Welch MJ. Indium-111-labeled leukocytes for the localization of abscesses: preparation, analysis, tissue distribution and comparison with Ga-67 citrate in dogs. J Lab Clin Med. 1977; 89:217-228. [PubMed: 401519]

2. Desai AG, Thakur ML. Radiolabeled blood cells: techniques and applications. Crit Rev Clin Lab Sci. 1986; 24:95-122. [PubMed: 2844472]

3. Thakur ML, Marcus CS, Kipper SL, et al. Imaging infection with LeuTech. Nucl Med Commun. 2001; 22(5):513-519. [PubMed: 11388572]

4. Kipper SL, Rypins EB, Evans DG, et al. Neutrophil-specific 99mTc-labeled anti-CD15 monoclonal antibody imaging for diagnosis of equivocal appendicitis. J Nucl Med. 2000; 41(3):449-455. [PubMed: 10716317]

5. Thakur ML, Marcus CS, Henneman P, et al. Technetium-99 m labeled anti-SSEA-1 antibody specific for human neutrophils: In vivo evaluation and assessment as an agent for imaging infectious diseases. J Nucl Med. 1996; 37:1789-1795. [PubMed: 8917176]

6. Rao PS, Pallela VR, Vassileva-Belnikolovska D, et al. A receptor-specific peptide for imaging infection and inflammation. Nucl Med Commun. 2000; 21:1063-1070. [PubMed: 11192713] 
7. Dockrell DH, Whyte MKB. Regulation of phagocyte lifespan in the lung during bacterial infection. J Leukoc Biol. 2006; 79:904-908. [PubMed: 16478918]

8. Simon HU. Neutrophil apoptosis pathways and their modifications in inflammation. Immunol Rev. 2003; 193:101-110. [PubMed: 12752675]

9. Fialkow L, Filho LF, Bozzetti MC, et al. Neutrophil apoptosis: a marker of disease severity in sepsis and sepsis-induced acute respiratory distress syndrome. Crit Care. 2006; 10:R155. [PubMed: 17092345]

10. Bratton DL, Fadok VA, Richter DA, et al. Appearance of phosphatidylserine on apoptotic cells requires calcium-mediated non-specific flip-flop and is enhanced by loss of the aminophospholipid translocase. J Biol Chem. 1997; 272:26159-26165. [PubMed: 9334182]

11. Penn DL, Kim C, Zhang K, et al. Apoptotic abscess imaging with $99 \mathrm{~m}_{\text {Tc-HYNIC-rhAnnexin-V. }}$ Nucl Med Bio. 2010; 37:29-34. [PubMed: 20122665]

12. Hanshaw RG, Lakshmi C, Lambert TN, et al. Florescent detection of apoptotic cells by using zinc coordination complexes with a selective affinity for membrane surfaces enriched with phosphatidylserine. Chembiochem. 2005; 6:2214-2220. [PubMed: 16276499]

13. Leevy WM, Gammon ST, Johnson JR, et al. Noninvasive optical imaging of staphylococcus aureus bacterial infection in living mice using a bis-dipicolylamine-zinc(II) affinity group conjugated to a near infrared fluorophore. Bioconjug Chem. 2008; 19:686-692. [PubMed: 18260609]

14. Leevy WM, Lambert TN, Johnson JR, et al. Quantum dot probes for bacteria distinguish Escherichia coli mutants and permit in vivo imaging. Chem Commun (Camb). 2008; 20:23312333.

15. Thakur ML, Lavender JP, Arnot RN, et al. Indium-111-labeled leukocytes in man. J Nucl Med. 1977; 18:1014-1021. [PubMed: 409745]

16. Leevy WM, Gammon ST, Jiang H, et al. Optical imaging of bacterial infection in living mice using a fluorescent near-infrared molecular probe. J Am Chem Soc. 2006; 128(51):16476-16477. [PubMed: 17177377]

17. Vinegar R, Traux JF, Selph JL, et al. Pathway to carrageenan-induced inflammation in the hind limb of the rat. Fed Proc. 1987; 46(1):118-126. [PubMed: 3100339]

18. Luker GD, Luker KE. Optical imaging: current applications and future directions. J Nucl Med. 2008; 49:1-4. [PubMed: 18077528]

19. Weissleder R, Ntziachristos V. Shedding light onto live molecular targets. Nat Med. 2003; 9:123128. [PubMed: 12514725] 


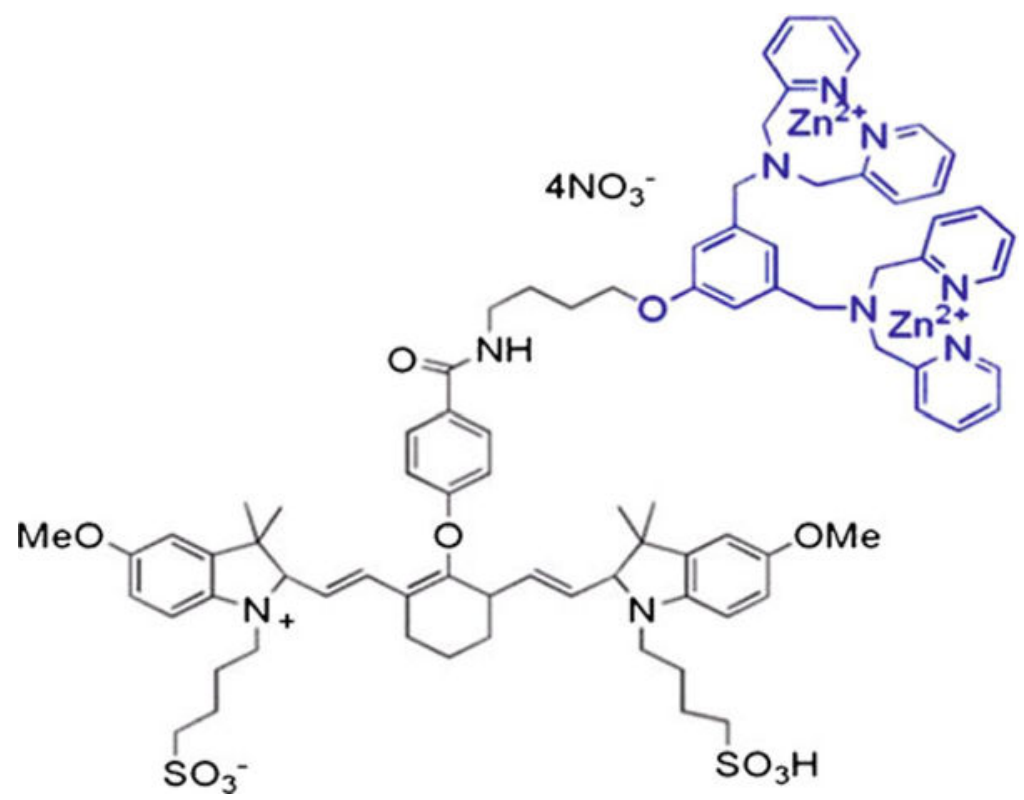

Fig. 1.

Structural formula of PSVue ${ }^{\circledR} 794$, a zinc(II) dipicolylamine probe. 


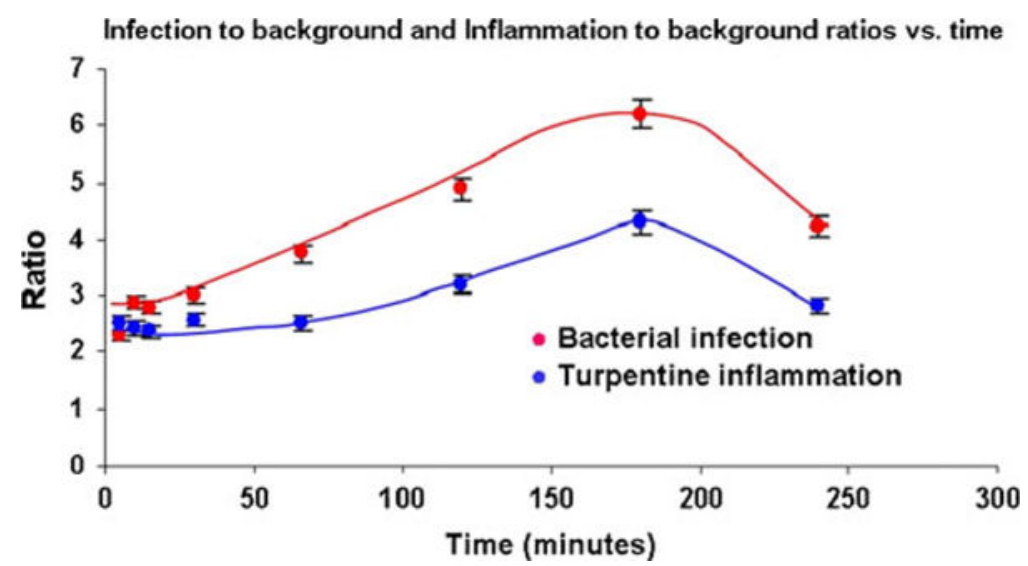

Fig. 2.

Bacterial infection to background and turpentine-induced inflammation to background image intensity ratios (averaged $n=5$ each) plotted as a function of time after the administration of the fluorophore. Data are obtained from the longitudinal images some of which are shown in Fig. 3. 


\section{Dynamic optical imaging of bacterial infection with PSVeu 794}

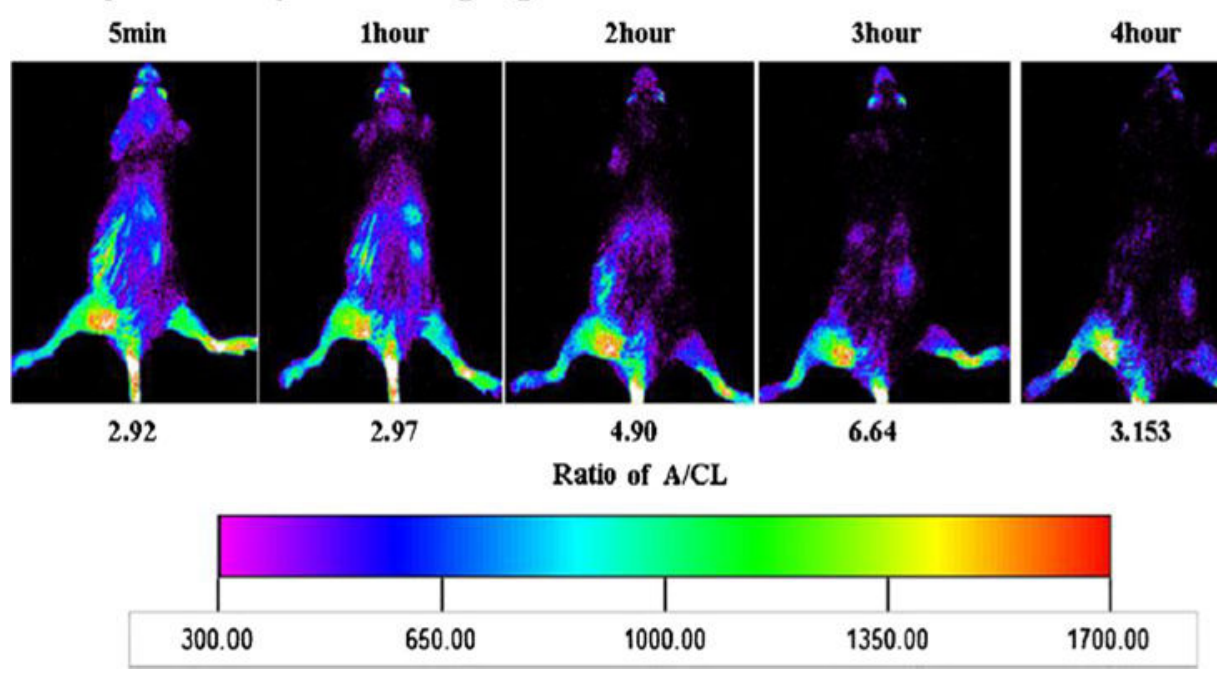

Fig. 3.

Dynamic optical images of one mouse bearing bacterial infection were acquired for $10 \mathrm{~s}$ at 5 min and at 1,2 , and $3 \mathrm{~h}$ post-tail vein injection of PSVue ${ }^{\circledR} 794$. Ratios of intensity in abscess to contralateral thigh (A/CL) are given. Declining uptake in liver and kidneys is visible as a function of time after injection. 


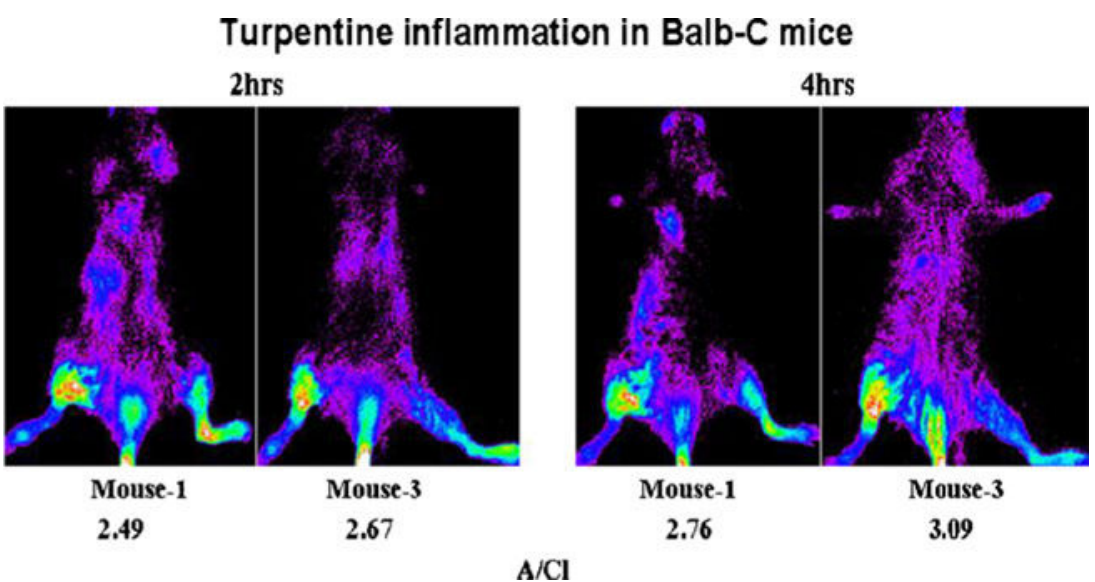

Fig. 4.

Posterior optical images of mice with turpentine-induced inflammation. Ten-second images were acquired at 2 and $4 \mathrm{~h}$ post-tail vein administration of $2.7 \mathrm{pmol}$ of PSVue ${ }^{\circledR} 794$. During intravenous injection, a small quantity of PSVue ${ }^{\circledR} 794$ was infiltrated, which is visible. The presence of the probe in the left leg was external contamination (x-ray images are not shown). 


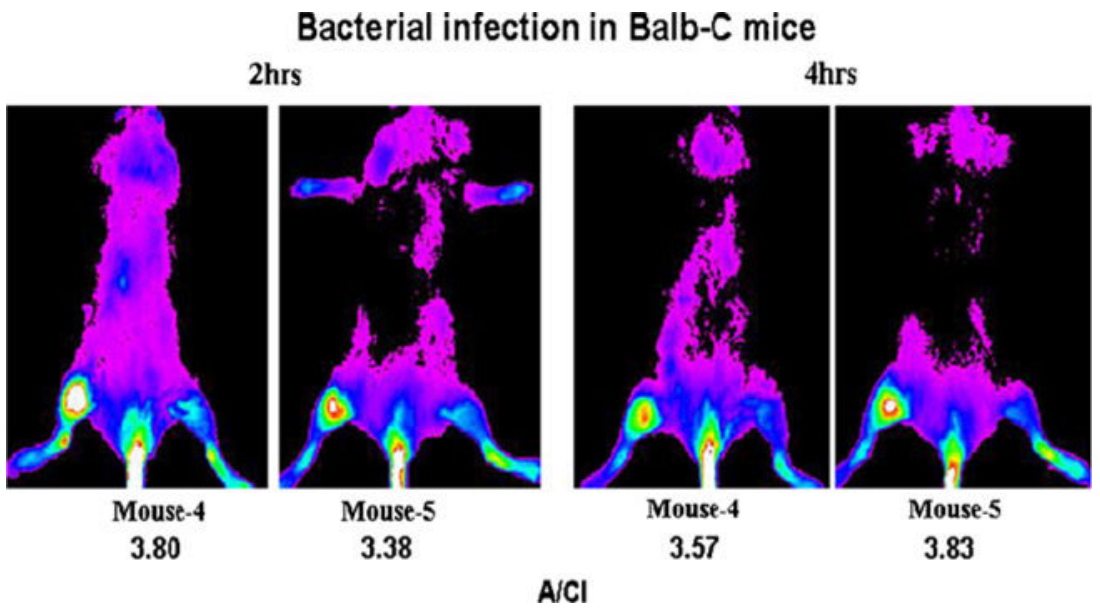

Fig. 5.

Posterior optical images of mice with bacterial infection in the right thigh. Ten-second images were obtained at 2 and $4 \mathrm{~h}$ post-intravenous administration of 2.7 pmol PSVue ${ }^{\circledR} 794$ (x-ray images are not shown). A small amount of the fluorophore infiltration of the probe in the tail during injection is visible. 

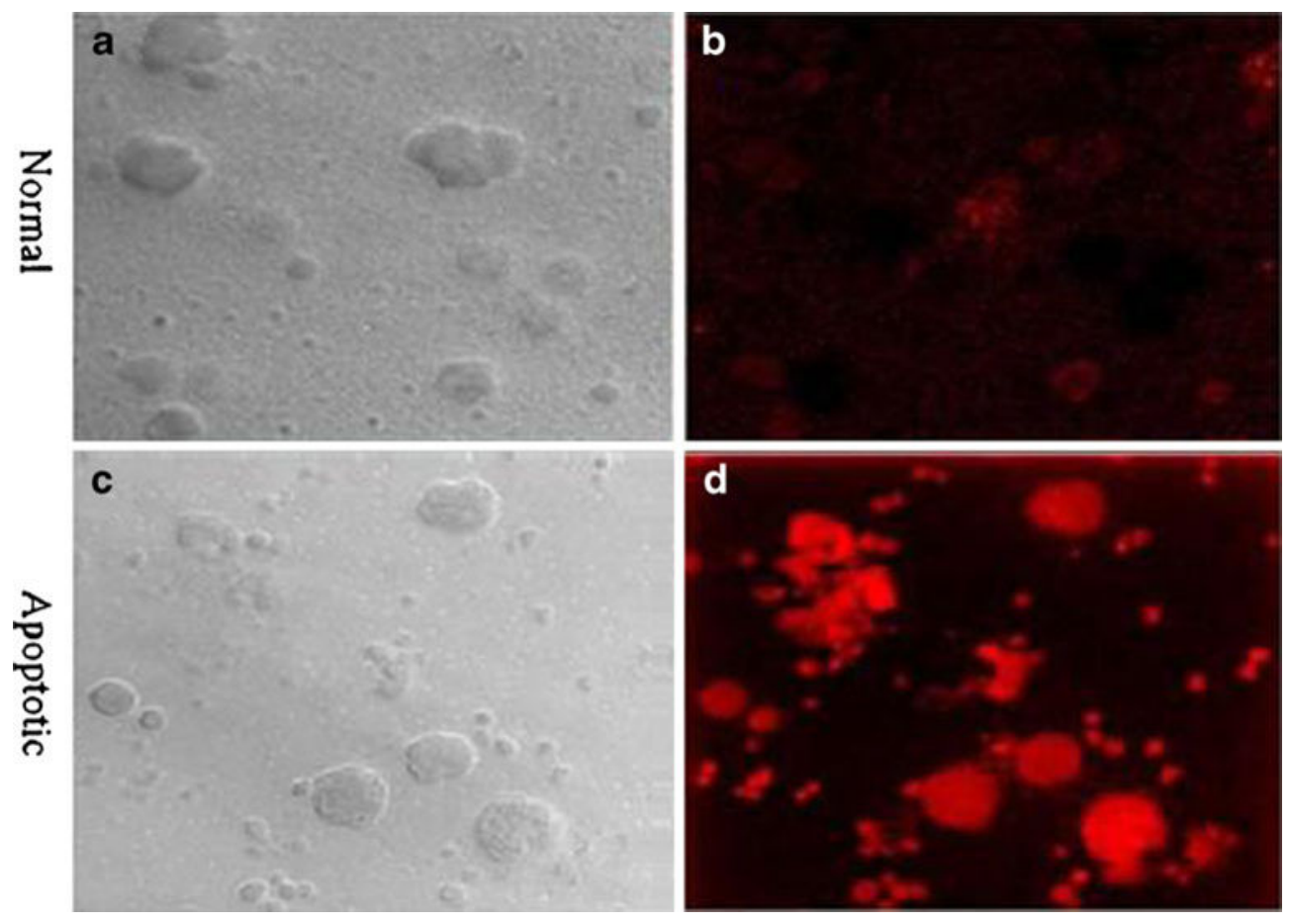

Fig. 6.

Confocal microscopic images of a normal and $\mathbf{b}$ PSVue ${ }^{\circledR} 794$-incubated normal white blood cells. Apoptotic white blood cells (c) with intense PSVue ${ }^{\circledR} 794$ fluorescence specific for phosphatidylserine $(\mathbf{d})(\times 63)$. 

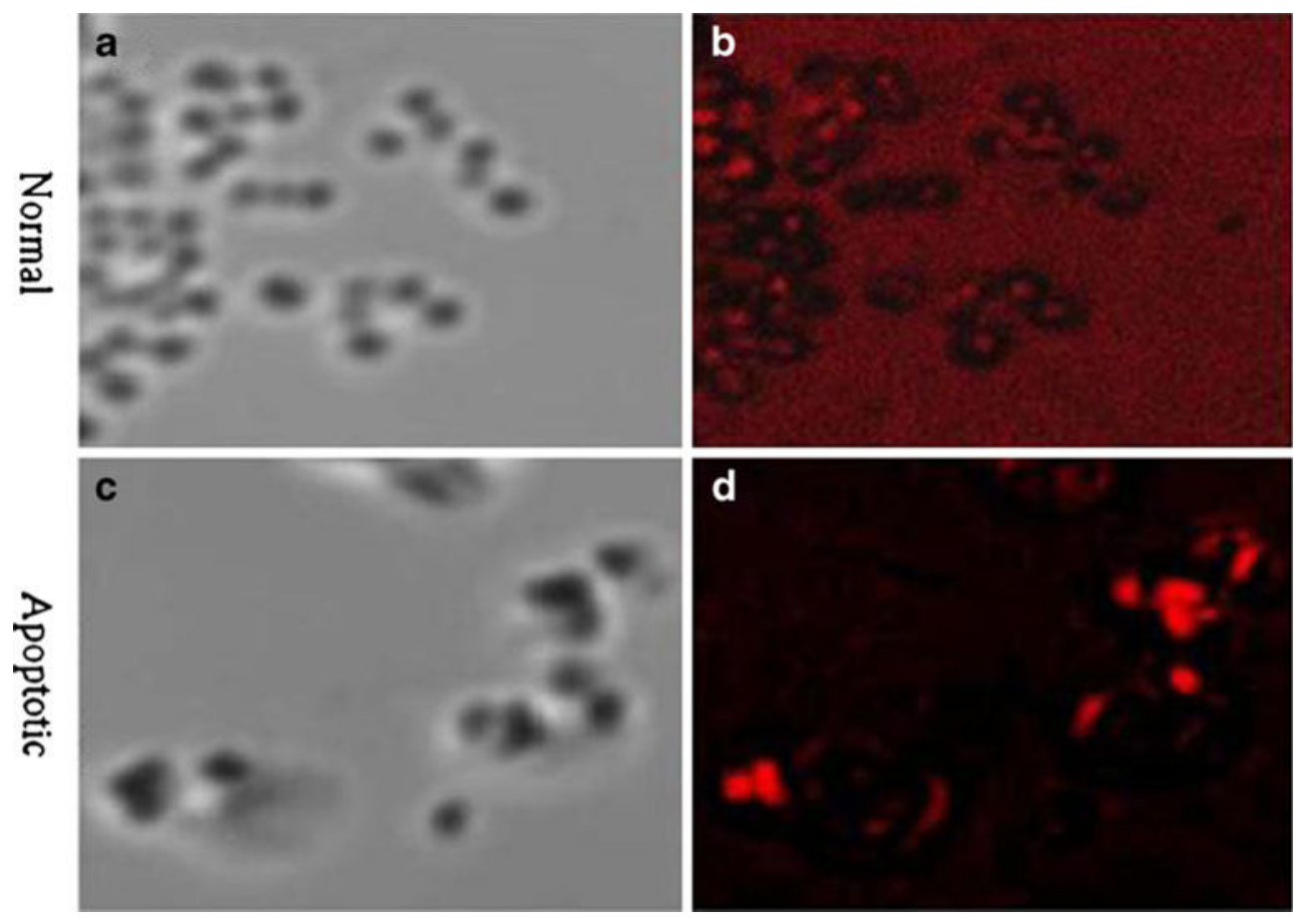

Fig. 7.

Confocal microscopic images of a normal gram-positive Staphylococcus lugdunensis. b In normal S. lugdunensis incubated with PSVue ${ }^{\circledR} 794$, fluorescence is detectable. c Confocal microscopic image of the microorganism. d Uptake of PSVue ${ }^{\circledR} 794$ in the apoptotic bacteria $(\times 400)$. 

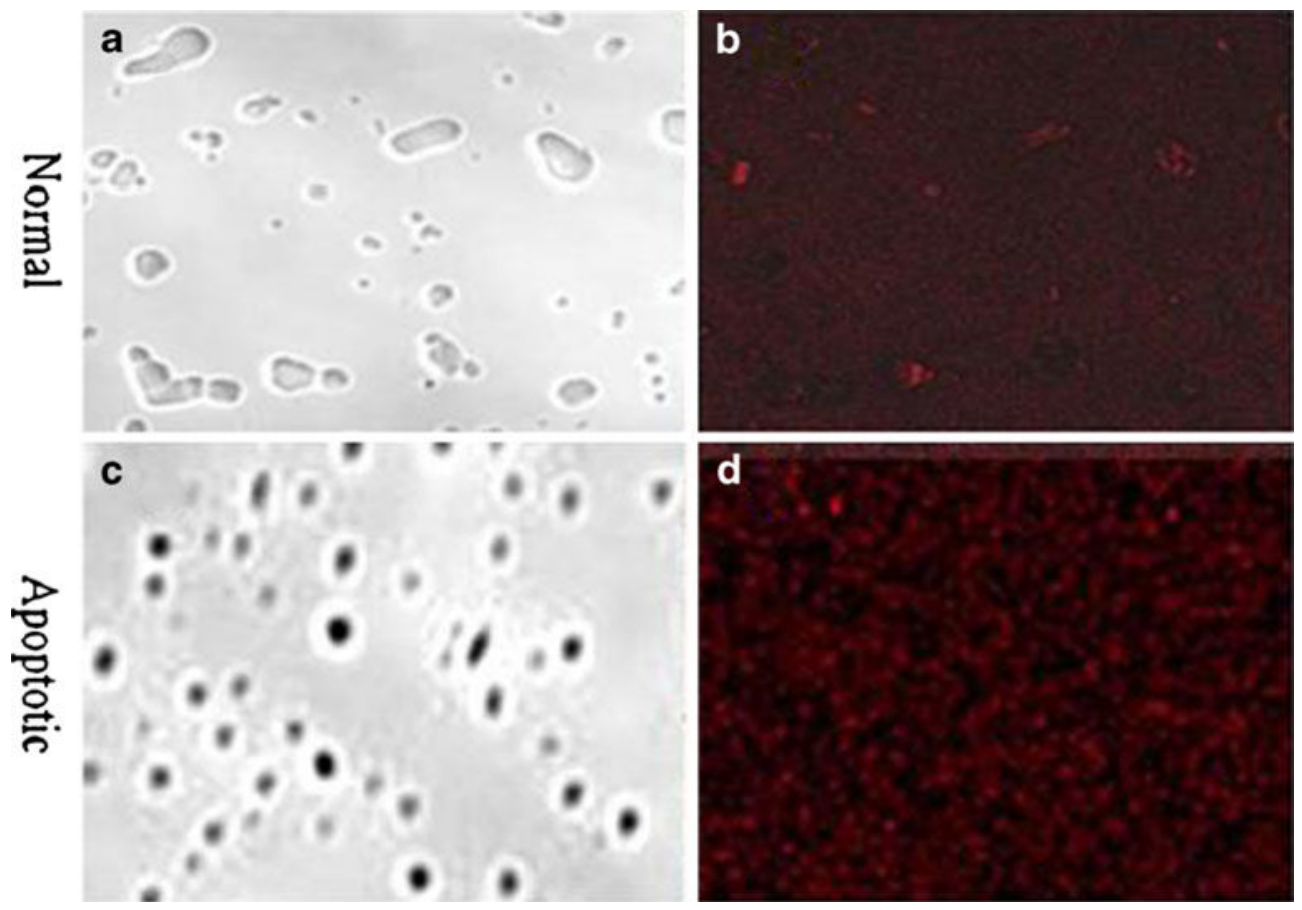

Fig. 8.

Confocal microscopic images of a normal $E$. coli gram-negative and $\mathbf{c}$ apoptotic $E$. coli. b Normal E. coli after incubation with PSVue ${ }^{\circledR} 794$. No bacterial fluorescence is detectable. d Poor fluorophore uptake in the apoptotic E. coli (ATCC-29213) $(\times 400)$. 